\title{
MUJERES INMIGRANTES ENTRE LA PRECARIEDAD FAMILIAR Y LA PRECARIEDAD LABORAL. ROLES DE GÉNERO Y DIVISIÓN SEXUAL DEL TRABAJO
}

\section{FEMALE IMMIGRANTS: BETWEEN PRECARIOUS FAMILY SITUATIONS AND PRECARIOUS WORK SITUATIONS. GENDER ROLES AND THE SEXUAL DIVISION OF LABOUR}

Carmen M. Benavides Zula (1)

Katerina L. Helguero Loayza (1)

(1) Trabajadoras sociales

Resumen: El presente artículo tiene como objetivo examinar los cambios producidos en los roles de género y la división sexual del trabajo en las familias transnacionales latinoamericanas afincadas en España, mediante una búsqueda, recopilación y análisis de bibliografía referente a la inmigración y el género. La parte central del artículo versa sobre los roles adoptados por las mujeres dependiendo de la fase del proceso migratorio en que se encuentren las familias transnacionales.

El artículo pretende así aportar una reflexión a la literatura ligada a las cadenas globales de cuidado que se establecen en el marco de la transnacionalidad. Del mismo modo, a lo largo de la investigación consultada se evidencia la escasa presencia de debates relativos a la transnacionalidad y la múltiple discriminación que sufren muchas de las mujeres inmigrantes dentro de la bibliografía seleccionada, ligada al tema que nos ocupa.

Palabras Clave: Roles de Género, Mujeres Inmigrantes Latinoamericanas, Discriminación Múltiple.

Abstract: The aim of this paper is to examine the changes occurring in gender roles and the sexual division of labour in transnational Latin American families living in Spain. The methodology involved the search for and the compilation and analysis of bibliographical sources on the topic of immigration and gender. The central part of the article looks at the roles adopted by women depending on the phase of the migratory process in which the transnational family finds itself at a given time.

The article contributes to the literature on the global care chains that arise in the context of transnationality. In addition, the research found a very limited presence of debate on the topic of transnationality and the multiple discrimination experienced by many of the immigrant women in the selected bibliography on this subject.

Key Words: Gender Roles, Latin American Immigrant Women, Multiple Discrimination.

| Recibido: 17.10.2011 | Revisado: 29.03.2012 | Aceptado: 03.04.2012 | Publicado: 01.05.2012 | 


\section{INTRODUCCIÓN}

El objetivo será explorar los cambios producidos en los roles de género y la división sexual del trabajo dentro de las familias transnacionales originarias de Latinoamérica que actualmente residen en España1. Como apuntan Camacho y Hernández (2005), "con relación a los cambios producidos en los roles de género se constata que en mayor o menor medida la ausencia de uno de los integrantes de la pareja ha obligado a mujeres y hombres a asumir nuevos roles y responsabilidades".

En la actualidad, una de las realidades fundamentales de la inmigración es su feminización. Como apunta Cristina Sánchez, "La feminización del flujo migratorio de América Latina y el Caribe hacia la Unión Europea, tiene vínculos con feminización de la pobreza, lo que se opera más destacadamente en países como República Dominicana, Ecuador y Colombia".

En concreto, en España desde finales de los noventa hasta la actualidad la población inmigrante latinoamericana no ha dejado de incrementarse, "las mujeres extranjeras han quintuplicado su presencia" y "la mayor feminización se aprecia entre nacionalidades latinoamericanas" (Rodríguez Jaume y Peresón, 2008). Es por ello que el artículo se centra en la inmigración femenina latinoamericana en España.

Existen diversos ámbitos de estudio en relación al género y la inmigración. El presente trabajo se decanta en primer lugar, por reflexionar sobre el ámbito laboral de las mujeres latinoamericanas en España. En segundo lugar, se hace una aproximación a la redefinición de los roles de género que se llevan a cabo dentro de las fases del proceso migratorio.

Es importante reflexionar sobre el aspecto económico y laboral, ya que encontramos que la mujer inmigrante está anclada en determinados nichos de empleo. "La capacitación y preparación de la mujer inmigrante no es tenida en cuenta a la hora de acceder a un trabajo. Nadie pregunta qué sabe hacer, qué preparación tiene; su nicho, su lugar en la sociedad europea es: el cuidado de niños, ancianos, y enfermos, la hostelería, el servicio doméstico, y la prostitución. La formación y preparación de estas mujeres no se corresponden en la mayoría de los casos a los trabajos que realizan" (Carrizo, Luberiaga, y Ronquillo, 2005). Varios autores atribuyen el que la mujer inmigrante latinoamericana siga encasillada y segregada laboralmente al tipo de estructura social, la cual cronifica los roles de género dentro del ámbito laboral. Un ejemplo de ello serían las cadenas globales de cuidado (Rodríguez Jaume y Peresón, 2008; Orozco, 2007).

Teniendo en cuenta todo lo anterior, la hipótesis que manejamos en este trabajo se basa en que el factor económicolaboral influye directamente en el rol que desempeña la mujer siendo este, en la mayoría de los casos, subordinado al del hombre. Dicho factor se ve influenciado por la estructura patriarcal desde la cual se estructuran las precarias condiciones en las que la mujer ingresa al mercado laboral. Asimismo, el hecho de ser inmigrante dificulta la inclusión de la mujer en el mercado laboral. De ahí la discriminación múltiple que sufre la mujer inmigrante. 
La estructura que seguirá el trabajo estará divida en cuatro partes. La primera parte consistirá en explicar la metodología utilizada. La segunda parte está constituida por el marco teórico en el que se encuadra el trabajo. En un tercer punto, se plasmarán los resultados, donde se hará una subdivisión de las fases del proceso migratorio por las que pasa la mujer y su familia. Por último, se incluirán las conclusiones a las que se ha llegado después de reflexionar sobre el tema elegido.

\section{METODOLOGÍA}

Para la elaboración de este trabajo se ha contado con las herramientas basadas en la búsqueda, recopilación y análisis de bibliografía referente al tema en cuestión. La revisión bibliográfica supone la "recopilación sistemática de la información publicada relacionada con un tema" (Universidad de Derby 1995). Y en este caso, a fin de realizar la misma, la base principal es el análisis de fuentes documentales secundarias y terciarias. Sin embargo en algunos casos se ha tenido que acudir a fuentes primarias originales para profundizar en la información relevante en cuanto al tema que nos ocupa.

El proceso que se ha seguido a fin de llevar a cabo la revisión de bibliografía ha requerido de las siguientes etapas:

1. La estrategia de búsqueda. En vista de que en principio el tema de la inmigración y el género posee diversos enfoques se realizó una búsqueda basada en los términos clave tales como inmigración y género, familias transnacionales, inmigración latinoamericana, etc. Así también, la búsqueda por autores que se citan con frecuencia condujo a varias investigaciones publicadas acerca del tema. Y como tercera estrategia se señala el examinar la bibliografía a la que hacen referencia los trabajos que más se acercaban a las variables del tema y que se correspondían en mayor medida con los objetivos fijados.

2. El criterio de selección que se ha seguido teniendo en cuenta los objetivos de la revisión ha sido el título de la publicación, autores de credibilidad y experiencia en el tema y, por último, analizando el resumen con el fin de distinguir si los resultados del trabajo o el estudio se aplican al tema elegido (Abad, et al, 2003).

3. A fin de organizar la información se han llevado a cabo diversos mapas conceptuales donde plasmar de una manera gráfica y sintética los puntos de interés del artículo para que resulte un aprendizaje eficiente y significativo (Martín Martínez et al, 2006). Se recalca esta fase en el proceso creativo del trabajo puesto que el hecho de poder visualizar, estructurar, organizar y representar la información contribuyó a la "administración, resolución de problemas y planificación organizacional así como a la toma de decisiones” (Soria et al, 2007).

4. Como paso último antes de redactar el artículo se estima adecuado elaborar una tabla de análisis y síntesis después de realizar el mapa mental. En esta se incluye la información esencial de cada estudio y artículo de acuerdo al objetivo del trabajo para poder llevar a cabo una comparación (Burns y Grove, 2004). De esta manera la tabla facilitó la identificación de los hallazgos que son comunes en los diferentes estudios y a comparar y contrastar los resultados de varios trabajos. 
El proceso de análisis bibliográfico en cuanto a la inmigración femenina en España y análisis similares, fue el punto de partida hacia la confección del enfoque adoptado en cuanto al tema y que podría ser tomado como un primer paso para llevar a cabo un estudio empírico del tema.

\section{MARCO TEÓRICO}

En primer lugar, se explicarán algunas definiciones que resultarán fundamentales para entender la exposición del tema en cuestión. En relación al ciclo migratorio, Dassen sostiene que es "el conjunto de procesos por el cual las poblaciones que pertenecen a un espacio económico periférico entran, se establecen y se implantan en el espacio de un Estado-nación que pertenece a los polos centrales de la economía capitalista" (Cachón, 2002).

Este concepto pone de manifiesto el factor económico que en muchos casos será una variable decisiva a la hora de emprender el proceso migratorio. Dentro de los procesos migratorios se encuentra el modelo de migración transnacional, el cual surge en la década de los noventa. Este modelo plantea que los espacios de la migración se están modificando "siendo sus cimientos las densas redes de comunicación -instrumentales y simbólicas- que vinculan cotidiana y permanentemente a las comunidades de origen y destino" (Ariza, 2002).

La sociología de las migraciones ha acuñado el término de transnacionalismo, que hace referencia a la existencia de nexos sólidos entre los lugares de origen y destino de los inmigrantes. Surgen de esta forma las familias transnacionales, las cuales se definen según Bryceson y Vuorela (2002) como "aquella familia cuyos miembros viven una parte o la mayor parte del tiempo separados los unos de los otros, siendo capaces de crear vínculos que permiten que sus miembros se sientan parte de una unidad y perciban su bienestar desde una dimensión colectiva, a pesar de la distancia física" (Bryceson y Vuorela, 2002).

Esta nueva tipología de familia participa en lo que Amaia Orozco denomina cadenas globales de cuidado. "Las cadenas globales de cuidados son cadenas de dimensiones transnacionales que se conforman con el objetivo de sostener cotidianamente la vida, y en las que en los hogares se transfieren trabajos de cuidados de unos a otros en base a ejes de poder, entre los que cabe destacar el género, la etnia, la clase social, y el lugar de procedencia" (Orozco, 2007).

Dado lo anterior resulta fundamental el definir lo que se entiende por género, optando por la definición que ofrece el Instituto de la Mujer según la Organización de Naciones Unidas (ONU), donde se dice que el género es "la forma en que todas las sociedades del mundo determinan las funciones, actitudes, valores y relaciones que conciernen al hombre y a la mujer. Mientras el sexo hace referencia a los aspectos biológicos que se derivan de las diferencias sexuales, el género es una definición de las mujeres y de los hombres, construido socialmente y con claras repercusiones políticas. El sexo de una persona es determinado por la naturaleza, pero su género lo elabora la sociedad".

Y dentro de las familias inmigrantes es importante hablar sobre lo que son los roles y, más concretamente, los roles de género. Según Glòria Pial Marcet (1993), "entendemos por rol el conjunto de expectativas compartidas acerca de la conducta de quienes ocupan posiciones específicas en una 
estructura social". Como indica el Instituto de la Mujer, los roles de género son "el conjunto de tareas y funciones que realizan mujeres y hombres según lo que cada sociedad les asigna. Son comportamientos y conductas que se aplican artificialmente, como un cliché, en función del sexo de las personas".

No menos importante resulta el hecho de que las mujeres inmigrantes sufren una discriminación múltiple: por ser mujeres, por ser inmigrantes y por pertenecer a un estatus social generalmente inferior. Esta situación supone una intersección de factores (sexo, raza y el estatus social) que dan lugar a una remarcada discriminación. A este respecto, Carlota Solé (2008) defiende que "Ser mujer, de clase trabajadora y de origen inmigrante, o de una nacionalidad determinada, supone experimentar diversas formas de diferencia y de discriminación".

Por otro lado, Martínez Alarcón (2003) explica que "la mujer inmigrante no solo sufre la discriminación económica a la que se ven sujetos todos los inmigrantes, en concreto los pobres sino que, además, a ello se añade una discriminación de género por el hecho de ser mujer". Asimismo, el Instituto de la Mujer sostiene que "la discriminación múltiple está relacionada con la conexión entre el género y factores como la etnia, la edad, el estatus socioeconómico, la orientación sexual, la diversidad funcional, la localización geográfica, el nivel educativo o modelos de socialización en el país de origen".

Dentro de este contexto, encontramos que persiste una "ideología de género" tanto en la división sexual del trabajo como en las relaciones de parentesco (Gregorio, C. y Ramírez, A., 2000) por lo que resulta interesante analizar los roles que juegan las inmigrantes latinoamericanas durante todo el proceso migratorio y cómo en función de esos roles se forman los nichos de trabajo en los que están encasilladas.

\section{RESULTADOS: SITUACIÓN LABORAL DE LA MUJER INMIGRANTE LATINOAMERICANA}

Como se sostiene en las hipótesis anteriores, uno de los factores que incide en el rol de la mujer inmigrante es el económico laboral. Dicho factor está caracterizado por estructuras de discriminación propias del mercado de trabajo. De esta manera se describen, por ejemplo:

1. Las prácticas discriminatorias como actitudes y discursos que legitiman la dominación y que parecen jugar un rol bastante importante puesto que tales diferencias pueden producir desigualdades en distintos campos, entre ellos el del empleo (IMSERSO, 1999).

2. La discriminación basada en la diferencia nacional-cultural, en otras palabras, el racismo, o nuevo racismo como sostienen algunos autores, basado no en la raza sino más bien en las diferencias culturales; al fin y al cabo un discurso que legitima la discriminación y la diferenciación de autóctonos como superiores e inmigrantes como inferiores.

3. Discriminación por sexo en el trabajo: que aunque parece afectar también a la población española sin embargo, como afirma Cachón, resulta un agravante más en el caso de los inmigrantes (Cachón, 2004).

4. Mucho más relevante resultan los estereotipos y prejuicios que tienen que ver con el género y con la etnia, puesto que estos resultan en salarios más bajos, menor estabilidad laboral, etc., independientemente de su formación o capacidad, solo por el hecho de ser inmigrante, sufriendo así discriminación y situándose en los huecos laborales que rechazan en muchas 
ocasiones los autóctonos. Un ejemplo de la influencia de los estereotipos se observa en que las mujeres latinoamericanas son en su mayoría demandadas para servicios domésticos internos ya que se las cree "dóciles y cariñosas"; mientras que en el caso de trabajos por horas las autóctonas son más demandadas (se piensa que son más rápidas en su trabajo y más limpias). Pero, en general, los clientes prefieren a las trabajadoras extranjeras porque creen que van a ahorrarse dinero ya que presuponen que se les pagará menos por sus servicios y que van a ser menos exigentes y reivindicativas que las autóctonas (Cachón, 2004).

Todos estos elementos discriminatorios se traducen en precariedad laboral, desigualdad salarial y nichos de empleo, los cuales se detallan a continuación.

\subsection{PRECARIEDAD LABORAL}

Pese a las restricciones que se aplican en las políticas migratorias, resulta muy interesante para muchos (empresas y particulares) la inmigración ilegal, ya que esta es apreciada como una fuerza de trabajo muy barata y maleable al antojo. A su vez, esta precariedad laboral caracterizada por unas pésimas condiciones de trabajo y unos bajísimos ingresos provocan en la población autóctona un sentimiento de rechazo. Esta circunstancia permite comprender por qué hay una mayor proporción de mujeres inmigrantes que ocupan los estratos más devaluados del mercado laboral femenino. Así lo señala Parella (2002) cuando dice, en palabras de Anderson (2000) que "las condiciones legales bajo las que estas mujeres desempeñan el servicio doméstico permite etiquetar esta actividad como una nueva forma de esclavitud".

Esta esclavitud se da tanto en las empresas privadas como en el interior de las familias autóctonas. Las empresas que ofertan cuidados, como lo pueden ser las empresas de servicios de ayuda a domicilio así como las residencias de ancianos suelen imponer lamentables condiciones laborales para las empleadas. Del mismo modo, en el interior de las familias contratantes de servicios de cuidados se ofrece poca remuneración, descansos ínfimos y todo ello, por lo general, de manera informal ya que no se les suele hacer un contrato de trabajo.

\subsection{DESIGUALDAD SALARIAL}

La discriminación y las desigualdades que se hacen hacia las mujeres con respecto a los hombres se reflejan, entre otras cosas, en la diferencia de salarios que perciben, así lo recalca Parella (2002) "por un lado, están peor pagadas las actividades atribuidas al género femenino que las atribuidas al género masculino; por otro, es habitual que las mujeres reciban menos salario que los hombres en una misma categoría laboral -a igual formación, dedicación y ejecución- y que tengan menores oportunidades de promoción profesional”. Esta situación se ve acentuada en las personas inmigrantes.

Según el último Informe sobre Igualdad Salarial de UGT de 2011 "las mujeres inmigrantes sufren una brecha salarial importante con respecto a los hombres inmigrantes, al trabajar en sectores más precarizados y en la economía informal" (UGT, 2011). 
Resulta importante lo que dice este informe en palabras de la Organización Internacional del Trabajo cuando sostiene que "la igualdad de remuneración no se alcanza logrando que hombres y mujeres ganen lo mismo, ni tampoco cambiando el tipo de trabajo que realiza la mujer, sino que está en subsanar la depreciación de los empleos ocupados por mujeres y en retribuirlos en función de su valor real, así como en asegurar una redistribución de roles entre hombres y mujeres" (UGT, 2011).

\subsection{NICHOS DE EMPLEO}

En los últimos años la mujer española ha alcanzado un mayor nivel educativo que le ha permitido acceder a mejores oportunidades laborales. Esto se traduce en que dichas mujeres se emplean en trabajos remunerados fuera del hogar, abandonando los trabajos reproductivos propios del ámbito privado, los cuales están catalogados como "actividad servil". De esta forma, dejan una vacante en las tareas que habitualmente tienen asignadas en el hogar y que ocupa la mano de obra inmigrante. Como sostiene Parella (2002) en palabras de Gregson y Lowe (1994) "la liberación' de algunas mujeres se produce a costa de la 'opresión' de otras".

Estos nuevos empleos están caracterizados por la precariedad laboral, el desprestigio social, ínfimos salarios, y por la falta de regulación laboral. Sin embargo, todo esto ha sido creado como respuesta al vacío que deja la mujer española al dejar de lado las labores reproductivas. Algunas de las respuestas que se han dado ante este fenómeno son las siguientes:

Por parte del Estado, tal y como señala Elin Peterson en palabras de Anthias y Lazaridis, "la oferta de trabajadoras domésticas ha sido promovida por el propio Estado, ya que ha habido políticas dirigidas a la entrada de inmigrantes para que se insertaran en el trabajo doméstico a través de un sistema de cuotas" (Peterson, 2009). Estas políticas han servido, por un lado, para facilitar a la mano de obra inmigrante el acceso al mercado de trabajo. Por otro, también encasillan a las mujeres inmigrantes en las mismas labores que muy probablemente realizaban en su país de origen y que en el de destino siguen reproduciendo.

Grandes estrategias para mejorar el rendimiento de las empresas se gestan a costa de reducir costes en los sueldos y en las condiciones de trabajo que se ofrecen a las trabajadoras inmigrantes. Este es el motivo por el que asistimos a una gran demanda de mano de obra femenina (como por ejemplo en el servicio doméstico, el cuidado de niños y ancianos así como en la hostelería y la prostitución) donde no se exige una gran cualificación. Sin embargo, se ofrece una gran explotación ya que son puestos de trabajo mal pagados y donde no se brindan derechos a las trabajadoras. Esta variada oferta de trabajos precarios se sitúa en las últimas posiciones de la estructura ocupacional. Es en este punto donde una de las razones para decir "sí" a esta situación es la inevitable comparación que hacen las mujeres inmigrantes de su situación con respecto al país de origen. Por muy mal que estén aquí, siempre será mejor que en su tierra natal. Así lo reflejan Silvia Carrizo et al. (2006) cuando señalan que "la posibilidad de movilidad social del inmigrante en el país de acogida es prácticamente imposible, pero ser pobre se valora positivamente, visto desde la perspectiva de la situación en su país. La trabajadora doméstica de jornadas interminables de siete días de la semana, al compararse con la mujer que conoce en el país de origen siempre se va a ver mejor". 
En concreto, cuando hablamos de nichos de empleo de la inmigración femenina latinoamericana, se observa que esta desempeña actividades relacionadas con los típicos roles femeninos que se desarrollan en su ámbito privado. Así, no es difícil ver a muchas de estas mujeres desempeñar papeles de cuidadoras de niños, enfermos o ancianos. A este respecto Sònia Parella (2002) sostiene que "el servicio doméstico constituye, prácticamente, la única oportunidad laboral para las mujeres de origen inmigrante en la sociedad receptora, con independencia de cuál sea su nivel de estudios y su experiencia laboral previa. Esta posición es resultado de la confluencia de la 'triple discriminación' (por clase, género y etnia) que padece la mujer inmigrante y que la relega al escalafón más bajo de la estructura ocupacional".

En cuanto a los trabajos relacionados con el cuidado que desempeñan las mujeres inmigrantes hablaremos del fenómeno de las cadenas globales de cuidados ${ }^{2}$ que plantea que recae sobre las mujeres las tareas de cuidados. Al liberarse de estas tareas por incorporarse al mercado laboral, dichos espacios son cubiertos por mujeres inmigrantes. Estas a su vez encuentran así un modo de ganarse la vida pero a costa de dejar también un hueco en la provisión de cuidados en el seno de sus hogares. El tercer eslabón de la cadena lo conforman las madres, hijas mayores, tías, etc. de la mujer inmigrante, las cuales tienen encomendada la labor de cuidar de los hijos de las mujeres inmigrantes. De esta manera se van articulando los eslabones que conforman las cadenas globales de cuidados. Estas cadenas tienen, como es de esperar, un perfil claramente femenino, donde la figura paterna apenas se percibe como responsable de otorgar los cuidados que ya no puede procurar la mujer en su familia. La conformación de estas cadenas se rige por los roles de género, los cuales responden a unos patrones rígidamente patriarcales.

Algunos autores señalan el hecho de que las redes sociales migratorias influyen en que la mano de obra inmigrante se concentre en nichos laborales, de hecho Parella (2002) sostiene que "las redes migratorias no solo intervienen en la decisión de emigrar sino también en la conexión entre la oferta de fuerza de trabajo en el país de origen y la demanda en la sociedad de destino".

\section{ANÁLISIS Y DISCUSIÓN}

"La 'división sexual del trabajo' supone el reparto social de tareas o actividades según sexo-género", Uribe-Echevarría (2008). Es decir, asistimos a una diferenciación en la inserción de hombres y mujeres en cuanto a la división sexual del trabajo. Por tanto, se hará una distinción entre el espacio reproductivo y el de la producción social. Dentro del espacio de reproducción se observa que la mujer está segregada en tareas domésticas y de cuidado. Por otro lado, dentro del espacio productivo, se verá una segregación ocupacional puesto que las mujeres estarán agrupadas en determinados sectores de actividad, ocupaciones y puestos de trabajo.

Sin embargo, se toma en cuenta que la división del trabajo "es específica de cada cultura y época en particular, por lo que no se trata de una división social del trabajo universal y rígida, sino que posee un carácter flexible" y podrá estar determinada por las "circunstancias socioeconómicas de una comunidad, región o grupo familiar". En el caso concreto de España se comprueba que la división sexual 
del trabajo ha ido evolucionando a lo largo de los años, llegando la mujer española en su intento de superar los roles de género (en los que estaba inmersa hasta ocupar nuevas posiciones en el mercado laboral) a relegar a un segundo plano su papel de cuidadora en la familia. En este escenario, se encuadra el papel de la mujer inmigrante la cual, como se ha mencionado anteriormente, se ocupa en los trabajos reproductivos a fin de ocupar los vacíos que deja la mujer española en su ámbito privado ${ }^{3}$.

Hay un contraste entre el efecto "escapada" que hace la mujer española con respecto a los roles que habitualmente se le atribuyen en el ámbito privado, y el confinamiento al que se ve sometida la mujer inmigrante durante todo su proceso migratorio. Por tanto, en este apartado se reflexiona sobre qué conflictos se perciben en las fases del proceso migratorio para las familias desde una perspectiva de género. Según señalan Ana María Rivas y Herminia Gonzálvez (2009), "el análisis de las relaciones y prácticas de género en unidades familiares transnacionales hace visible la diversidad de arreglos familiares que se establecen en la etapa previa, y que se desarrollan o mantienen durante y después de la migración". Con este fin, se han tomado en cuenta principalmente dos estudios ${ }^{4}$ cuyo objetivo son las mujeres inmigrantes en España para analizar la redefinición de los roles en las relaciones de género de las mujeres inmigrantes y sus familias.

Según estos estudios las fases del proceso migratorio serían las siguientes:

\section{Antes de emigrar}

A diferencia de lo que muchas veces se ha considerado de un modo erróneo, el proceso migratorio se inicia desde antes de emigrar. Como señala Adelmalek Sayad (citado en Herrera, 2002) "antes de ser inmigrante se es emigrante”. (Bertino Mena, Arnaiz Adrián, y Pereda Sagredo, 2006). Es así como la situación de origen de la mujer inmigrante influirá en gran medida en la forma en que se vivirá la migración en el destino. La emigración en la mayoría de los casos es parte de una planificación familiar, o bien se debe a la incapacidad de los varones para cumplir con el rol tradicional de proveedores económicos, lo que se denomina erosión del papel del varón como proveedor económico (Posso Quiceno y Urrea Giraldo, 2007). En el primer caso la mujer, siendo parte de un "grupo doméstico", se ve subordinada a la autoridad masculina y su decisión de emigrar es considerada como una estrategia más del grupo doméstico para la supervivencia dentro de las clases sociales más desfavorecidas (Gregorio, 1997). En el segundo caso, siendo las mujeres quienes ya en su país de origen soportaban la mayor parte de la carga en términos económicos para el sostenimiento de la familia, toman la decisión de emigrar ligada a una estrategia de supervivencia, especialmente por la condición de maternidad y el contexto familiar (Martinez Pizarro, 2003).

Sin embargo, la mayoría de autores, afirman que la decisión de la migración no es tomada libremente por la mujer sino más bien es fruto de una decisión abstractamente familiar, por lo que se liga grupo doméstico y redes migratorias. Esto constata cómo se imponen los roles de género tradicionales

3 Esto no significará que la mujer española se desvincule totalmente de los trabajos de reproducción.

4 Estudio n. 1: Investigación "Mujeres migrantes con menores a cargo en país de origen" Estudio cualitativo sobre variables multidimensionales y efectos psicosociales en el país de destino (Navarra).

Estudio n. 2: Proyecto de investigación "Redes familiares transnacionales, los reacomodos en los ámbitos productivo y doméstico entre hombres y mujeres en las familias de emigrantes colombianos en España". 
dejando a la mujer en un segundo plano. A este respecto, Jiménez (1998) afirma que “...una mujer que migra sola no tiene por qué tener un alto nivel de poder de decisión, ya que puede hacerlo por imposición familiar para, por ejemplo, diversificar los riesgos de la economía doméstica".

Falicov (2006) señala que "cuando emigran, se arriesgan a ser juzgadas por el discurso social patriarcal que enturbia la reputación de las mujeres independientes”.

\section{Una vez ya en España}

Cuando la mujer llega al país de acogida se ve sometida a largas jornadas de trabajo para, como nos dicen Posso y Urrea (2007), poder compensar los ínfimos salarios a los que pueden acceder. En este momento la mujer toma un nuevo rol dentro de la familia ya que comienza a ser la proveedora principal. Destacable es lo que sostienen Bertino Mena et al. (2006), ya que en esta fase la mujer se ve sometida a un proceso de duelo por la pérdida de estatus, y por el sentimiento de haber abandonado a su familia. Todo esto junto con la obligación de la manutención del grupo familiar por medio de las remesas, lo que puede provocar en la mujer ansiedad y estrés, llegado incluso a experimentar sentimientos de culpabilidad no permitiéndose algún gasto "superfluo" que revierta en su propio bienestar. Lo que complica la posibilidad de relacionarse socialmente. Un ejemplo de esto es lo que nos dice una mujer de 43 años de origen colombiano:

"Todo lo que gano lo mando para allá. A mi cuñada se lo mando porque ella es la encargada de comprar los dodotis y las medicinas para mi mamá, y la comida de la casa, el arrendamiento, el colegio, todo (...) me repercute enviar, no tengo para transportes. Solo pago la habitación para no dormir en la calle. No tengo para comer a veces. El sueldo se divide en mi parte, mi madre y mi familia. La residencia de mi mamá vale 350 euros, más la medicina, con otros 300 se arregla mi familia”. (43 años, Colombia). (Martinez Pizarro, 2003).

Por tanto, después de la migración la mujer no deja de lado su rol de cuidadora, sino que lo agrega al nuevo rol de cabeza de familia. Esto supone que el hombre no suele tener una presencia mayor en las labores de reproducción que deja su mujer al emigrar, sino que sigue confiando a esta dichas tareas.

\section{Después de la reagrupación familiar}

Dentro de esta tercera fase podríamos subdividirla en dos, después de la llegada de la pareja, y después de la llegada de los hijos.

3.1. Llegada de la pareja. Después de que la mujer logra reunirse con su pareja en España, las decisiones en cuanto a la gestión del dinero cambian, puesto que al haber existido una anterior fase donde la mujer se ha empoderado y ha tomado nuevos roles, la relación de poder se modifica en muchos casos. Sin embargo, la mujer la mayoría de las veces está cansada de llevar la carga de ser la cabeza de familia. Consideran esta nueva circunstancia como una situación de relevo en la responsabilidad como proveedoras principales en la cual encuentran alivio. En muchos casos vuelven a su rol doméstico en el hogar complementado, por supuesto, por su jornada laboral reducida dentro del mercado de trabajo. Por tanto, la migración permite a los hombres recuperar su rol como proveedores principales, llegando a 
ocupar empleos mejor remunerados que los femeninos. Esta situación supone relegar a la mujer a un plano secundario. Es interesante descubrir en la investigación ${ }^{5}$ que muchas parejas jóvenes desarrollan un mayor equilibrio en las relaciones de poder. Esto se traduce en que ambos comparten actividades de la organización de los espacios internos de la casa, de la dieta alimenticia y de las actividades familiares. Sin embargo, esta idea no debe generalizarse.

De hecho, pese a que las mujeres suelen tomar parte en las decisiones familiares, las resoluciones siguen guiadas por las pautas de género y desde estructuras patriarcales. Sucede así que el hombre se encarga de la mayoría de los gastos como "el alquiler, los servicios, etc..." mientras que la mujer se ocupa de los gastos de "alimentación, vestuario" e incluso llega a pedirle ayuda a él para cumplir con su parte debido a las diferencias salariales que hay entre ambos.

3.2. Llegada de los hijos: en el momento de la llegada de los hijos, normalmente después de un año o dos, la figura paterna suele encontrarse en una posición superior a la de la mujer al tener un trabajo más estable y mejor remunerado. En este periodo influirá la etapa de desarrollo en que se encuentren los hijos. Como señala Falicov, "el niño rencoroso o el adolescente rebelde pueden estar atravesando el shock cultural y la depresión relacionadas con la ambivalencia del inmigrante recién llegado. La madre puede estar viviendo con otro hombre ahora, o haber tenido un nuevo hijo".

Las consecuencias de estos efectos de dominación son tanto para la madre como para los hijos. Por una parte, las mujeres se sienten enteramente responsables del cuidado de sus hijos, sin elaborar en muchos casos ideas de reparto de roles que contribuyan a minimizar su sufrimiento y repartir la responsabilidad con sus parejas. Al duelo por la partida de su tierra que sufren las mujeres inmigrantes se une el duelo provocado por la lealtad que sienten hacia sus hijos e hijas, a quienes privan de todo su cuidado. La asimilación que hacen los menores de que es la madre quien les debe proporcionar los cuidados que necesitan los lleva a albergar un gran resentimiento hacia sus progenitoras, pues estas los dejan en el país de origen con otros familiares.

\section{CONCLUSIONES}

Este artículo ha debatido sobre cómo los roles de género se van modificando a lo largo de todo el proceso migratorio de la familia transnacional. Es así como se ha llegado a la conclusión, gracias a la literatura consultada en materia de familias transnacionales y roles de género, de que existen grandes diferencias entre los roles que ejercerá la mujer y el hombre. Esta situación se debe a una amalgama de factores que están construyendo el problema, como lo son el sistema patriarcal y la incorporación de las mujeres inmigrantes al mercado de trabajo, lo cual las configura como nuevas cabezas de familia. Esto las llevará a asumir, no sin conflictos, mayores cotas de poder en la organización de su tiempo y en la toma de decisiones frente a sus compañeros, muchos de ellos desempleados.

5 Proyecto de investigación "Redes familiares transnacionales, los reacomodos en los ámbitos productivo y doméstico entre hombres y mujeres en las familias de emigrantes colombianos en España". 
El papel de la mujer inmigrante dentro de la esfera reproductiva no se cuestiona en el ámbito privado y laboral, lo cual refuerza su papel en la esfera doméstica. Con independencia de la formación que esta pueda poseer los trabajos a los que está llamada están relacionados con labores de cuidado y limpieza. Pese al cambio de roles experimentado en el proceso migratorio, en todo momento asume el papel de cuidadora, asignado como inherente a su condición de mujer. Así, se configura toda una red de cuidado transnacional. La rigidez de los roles femeninos es difícilmente quebrantable debido a las estructuras patriarcales, lo cual evidencia la diferente presencia que ocupan hombres y mujeres en dichos cuidados.

El actual contexto de crisis económica que atraviesa el país plantea nuevos retos futuros ya que nuevas situaciones pueden influir a las familias transnacionales. Tal circunstancia supondría nuevas líneas de investigación sobre estas familias. Sería interesante analizar qué puede suceder en los roles de las mujeres inmigrantes que retornen a sus países de origen como consecuencia de la falta de empleo en España. Las preguntas que cabría elaborar pasan por averiguar si las mujeres volverían a un papel cuidador, otorgando a sus parejas el papel de cabezas de familia o si, por el contrario, gracias a sus experiencias vividas en España las familias adoptarían nuevos acuerdos en el marco de la corresponsabilidad tanto dentro como fuera del hogar.

También sería conveniente investigar qué sucede en las familias que, pese a sufrir los efectos de la crisis económica, deciden quedarse en España. No es difícil intuir que en tal contexto económico las mujeres intentasen contribuir a la economía familiar de manera mucho más pronunciada. En este caso, ¿adoptarán las mujeres inmigrantes por segunda vez el papel de cabezas de familia?, ¿afianzarían este papel después de superar la crisis o acaso serían relevadas una vez más por sus maridos al estabilizarse la economía española? Son temas que interesaría desarrollar en profundidad.

\section{AGRADECIMIENTOS}

Dedicamos este trabajo a nuestras madres. Mujeres inmigrantes que lucharon para sacarnos adelante. Por ellas, porque somos la cosecha de nuestras madres. Gracias por vuestra tenacidad, la cual nos ha servido de inspiración para realizar este trabajo.

\section{BIBLIOGRAFÍA}

Agrela Romero, B. (2004). La acción social y las mujeres inmigrantes: ¿Hacia unos modelos de intervención? Revista de Trabajo Social Portularia, 4, 32-42.

Ariza, M. (2002). "Migración, familia y transnacionalidad en el contexto de la globalización: algunos puntos de reflexión". Revista mexicana de sociología, 64, 4, 53-84.

Aroyo Romero-Salazar, L. (2009). Situación económica, laboral y social de las mujeres en el contexto de crisis económicas. Observatorio Mujer, Trabajo y Sociedad, 14-17.

Avellanosa, I. (2006). A mi abuela la llamo mamá. Adolescencia, migración y género. Revista de estudios de juventud, 81-91.

Bedoya, M. H. (2000). Mujer extranjera: una doble exclusión. Influencia de la ley de extranjería sobre las mujeres inmigrantes. Papers, 241-256. 
Bertino Mena, L., Arnaiz Adrián, V., y Pereda Sagredo, E. (2006). Factores de riesgo y protección en madres migrantes transnacionales. Redes, 91-109.

Bryceson, D., y Vuorela, U. (2002). La familia transnacional: Nuevas Fronteras Europeas y la Red Mundial. Nueva York: Oxford.

Cachón, L. (2002). La formación de la España inmigrante: mercado y ciudadanía. REIS, 95-126.

Cachón, L. (2003). Inmigrantes jóvenes en España. Sistema educativo y mercado de trabajo. Madrid: Injuve.

Cachón, L. (2004). Inmigrantes y mercado de trabajo. Índice. Revista de estadística y sociedad, 16.

Camacho, G., y Hernández, K. (2005). Cambio mi vida. Quito: CEPLAES.

Canevaro, A. (2006). Terapia Trigeneracional para las simbiosis de parejas. Redes.

Carrizo, S., Luberiaga, J., y Ronquillo, S. (2005). Mujeres inmigrantes: de la invisibilidad a la presencia. Guipuzcoa: Malenetxea.

Chirino, F. (2007). Género, remesas y desarrollo humano. Análisis sobre la relación entre el manejo de las remesas, los cambios en los roles de género y el desarrollo humano de las familias de las personas inmigrantes. Recuperado el 16 de mayo de 2011, de Centro Boliviano de Estudios Disciplinarios:

http/ccd-ckd.cebem.org/documents/ccd021/Trabajo_Fabiana_Chirino.pdf

Escrivá, Á. (2000). ¿Empleadas de por vida? Peruanas en el servicio doméstico en Barcelona. Papers, 327-342.

Fahy Bryceson, D., y Vuorela, U. (2002). La familia transnacional: nuevas fronteras europeas y las redes mundiales. Nueva York: Oxford.

Gimeno, A. (octubre de 2009). Familias Transnacionales Colombianas: el nivel de aculturación y vida familiar vista desde las dos orillas. Madrid: Tirant Lo Blanch.

Gonzálvez Torralbo, H. (2008). Relaciones familiares transnacionales entre bello y elche. Madrid: Universidad Autónoma de Madrid.

Gregorio, C., y Ramirez, A. (2003). ¿En España es diferente...? Mujeres inmigrantes dominicanas y marroquíes. Papers, 60, 257-273.

Jaes Falicov, C. (2006). Migración, pérdida ambigua y rituales. Redes.

López Bermejo, P., y Rojo, C. (junio, 2009). Mujeres inmigrantes en el contexto de la crisis. Observatorio Mujer, Trabajo y Sociedad, 45-48.

Martín Díaz, E. (1 de agosto de 2008). El impacto del género en las migraciones de la migración: mujeres, trabajos y relaciones interculturales. Recuperado el 16 de mayo de 2011, de Scripta Nova: http://www.v.edu/gocrit/sn/sn-270/sn-270-133.htm

Martínez Alarcón, M. L. (2003). El inmigrante irregular. Especial referencia a la mujer inmigrante objeto de explotación sexual. Revista Española de Derecho Constitucional, 265-282.

Martinez Pizarro, J. (2003). El mapa migratorio de América Latina y el Caribe, las mujeres y el género. Santiago de Chile: Publicación de las Naciones Unidas.

Moreno, J. (2006). Feminización del fenómeno migratorio ecuatoriano. Haol, 121-132.

Mujer, I. d. (2004). Guía para la incorporación de la perspectiva de género. Madrid: Instituto de la Mujer. Muñoz de Bustillo, R., e Ignacio Antón, J. (2009). De la España que emigra a la España que acoge: contexto, dimensión y características de la inmigración latinoamericana en España. América Latina hoy: Revista de ciencias sociales, 15-39.

Nash, M. (2006). Lo intercultural en acción, identidades y emancipaciones. CIDOB D'AFERS, 39-57. Orozco, A. (2007). Cadenas globales de cuidados. Santo Domingo: Naciones Unidas.

Oso Casas, L. (2008). Migración, género y hogares transnacionales. En J. García Roca, y J. Lacomba, 
La inmigración en la sociedad española. Una radiografía multidisciplinar (pp. 561-586). Bellaterra: Ediciones Bellaterra.

Parella Rubio, S. (2002). La internalización de la reproducción. La inserción laboral de la mujer inmigrante en los servicios de proximidad. Barcelona: Universidad Autónoma de Barcelona.

Parella, S. (2003). Mujer, inmigrante y trabajadora: la triple discriminación. Barcelona: Anthropos.

Pedona, C. (2006). Los cambios familiares y educativos en los actuales contextos migratorios ecuatorianos: una perspectiva transatlántica. Athenea Digital, 154-171.

Peterson, E. (2009). Género y Estado de bienestar en las políticas españolas. Asparkía, 35-57.

Pizarro Martínez, J. (2003). El mapa migratorio de América Latina y el Caribe, las mujeres y el género. CEPAL. Serie población y desarrollo, 5-89.

Poal Marcet, G. (1993). Teoría de la socialización diferencial mujer-varón. Madrid: Siglo XXI.

Poggio, S. (2007). La experiencia migratoria según género:salvadoreños y salvadoreñas en el estado de Maryland. La Aljaba Segunda época, 11-27.

Posso Quiceno, J., y Urrea Giraldo, F. (2007). La migración internacional y los cambios en las relaciones de género y estructuras de los hogares:la migración colombiana hacia España. Papers, 109-133.

Puyo, L. (2009). Acortando las distancias: el trabajo del vínculo afectivo en familias transnacionales. Documento disponible en PDF. http://www.avntfevntf.com/imagenes/biblioteca/Puyo\%20L.\%20 Tbjo\%203\%20online\%2008\%281\%29.pdf.

Rivas Rivas, A. M., y Gonzálvez Torralbo, H. (2009). Familias transnacionales colombianas. Transformaciones y permanencias en las relaciones familiares y de género. Madrid: Catarata.

Rodríguez Jaume, M. J., y Peresón, C. (2008). Globalización e inmigración latinoamericana en España. Una mirada a las familias transnacionales. http://rua.ua.es/dspace/bitstream/10045/12007/1/ Globalizaci\%C3\%B3n\%20e\%20inmigraci\%C3\%B3n\%20latinoamericana\%20en\%20 Espa\%C3\%B1a.\%20Una\%20mirada\%20a\%20las\%20familias\%20transnacionales.pdf.

Rodríguez Jaume, M., \& Jareño, D. (2009). Mujeres, patrones reproductivos y familias extranjeras en España a la luz de la encuesta de fecundidad de 2006. VI Congreso sobre las Migraciones en España. La Coruña, 17-19 de septiembre de 2009., (págs. 1-6). La Coruña.

Sánchez, C. A. (2006, julio.). Inmigración Latina en Europa Género y Ciudadanía: La Dialéctica Atracción Repulsión ante el Avance de la Globalización. Revista de Filosofía, 5, 1-32.

Sassen, S. (2003). Contrageografías de la globalización. Género y ciudadanía en circuitos transfronterizos. Madrid: Traficantes de sueños.

Solé Puig, C., Parella Rubio, S., Ortega Rivera, E., Pávez Soto, I., y Sabadí Brugués, M. (2008). Las trayectorias sociales de las mujeres inmigrantes no comunitarias en España. Factores explicativos de la diversificación de la movilidad laboral intrageneracional. Barcelona: Gedime.

UGT. (2011). Informe sobre Igualdad Salarial. Madrid: UGT.

Datos extraídos de internet:

Estadística, I.N. (4 de abril de 2011). www.ine.es. Recuperado el 6 de abril de 2011, de http://www. ine.es/prensa/np648.pdf.

Madrid, C.d. (2009). www.madrid.org. Recuperado el 14 de febrero de 2011, de http://www.madrid.org/es/Satellite?blobcol=urldata\&blobheader=application\%2 Fpdf\&blobheadername1=Con tentdisposition\&blobheadername2=cadena\&blobh eadervalue1=filename\%3DPLAN+INT.0912. pdf.\&blobheadervalue2=language \%3Des\%26site\%3DPortallnmigrante\&blobkey=id\&b. 
Manuel Cué Brugueras; Guillermo Díaz Alonso; Ana Gloria Díaz Martínez; Manuela de la C. Valdés Abreu (1996). Artículo de revisión. Recuperado el 22 de abril del 2011, http://bvs.sld.cu/revistas/ spu/vol34 4 08/spu11408.htm

Ministerio de Trabajo e Inmigración (2011). Extranjeros residentes en España con certificado de registro o tarjeta de residencia en vigor a 31 de diciembre de 2010. Principales Resultados. Recuperado en http://extranjeros.mtin.es/es/InformacionEstadistica/Informes/Extranjeros31Dici embre2010/index.html

Universidad de Valencia (2010) Proyecto Fin de Carrera. Capítulo 4: Búsqueda y revisión de la Bibliografía. Recuperado el 22 de abril del 2011, del sitio web de la Universidad de Valencia. http:// informatica.uv.es/iiguia/2000/IP/libro/chapter4cgc.pdf

Telemadrid (Coproductor) y Marina Seresesky (Dir.) (2011). Madres, 0,15 el minuto [video]. España.

\section{ANEXOS}

\section{Figura 1. Población extranjera por continentes y sexo en España}

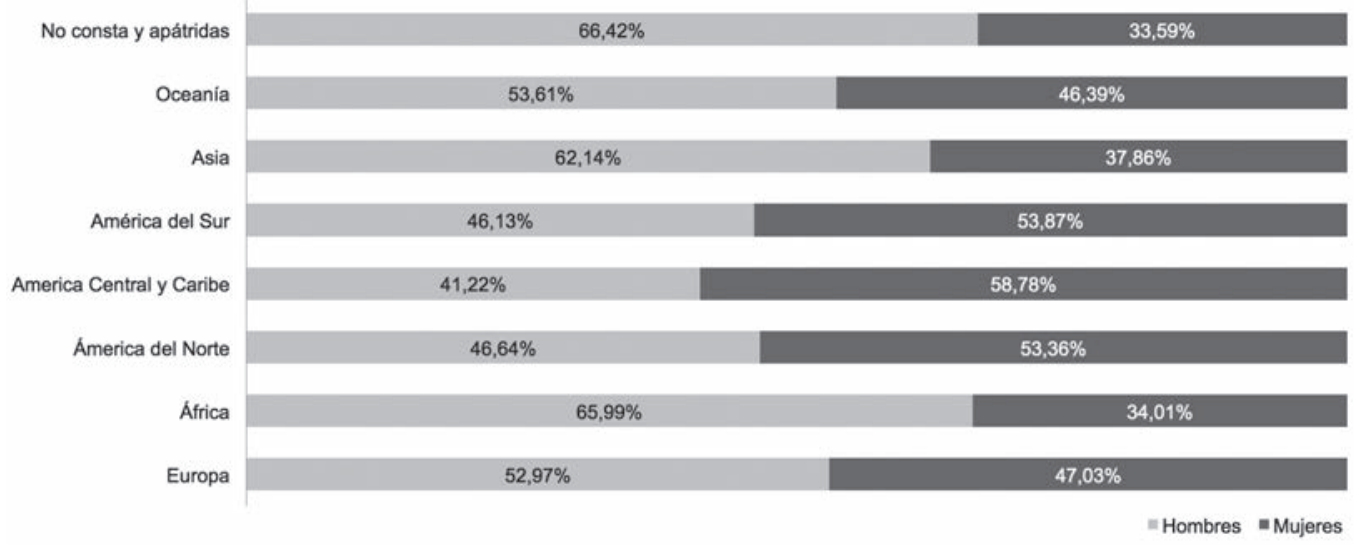

Fuente: Plan de Integración 2009-2012.

\section{Figura 2. Nacionalidades mayoritarias por sexo en España}

\begin{tabular}{|l|r|r|}
\hline & Hombres & Mujeres \\
\hline Rumanía & 391799 & 337168 \\
\hline Marruecos & 408338 & 236350 \\
\hline Ecuador & 206523 & 213587 \\
\hline Reino Unido & 178933 & 172986 \\
\hline Colombia & 124652 & 156053 \\
\hline Bolivia & 105611 & 134331 \\
\hline Alemania & 91267 & 89383 \\
\hline Italia & 92742 & 64693 \\
\hline Bulgaria & 84014 & 69650 \\
\hline Argentina & 72904 & 72411 \\
\hline
\end{tabular}

Fuente: Plan de Integración 2009-2012. 
Figura 3. Población extranjera por sexo y grupos de países

\begin{tabular}{|c|c|c|c|c|c|}
\hline & Total & Varones & $\begin{array}{c}\% \text { respecto al } \\
\text { total del grupo } \\
\text { de países }\end{array}$ & mujeres & $\begin{array}{c}\% \text { respecto al } \\
\text { total del grupo } \\
\text { de países }\end{array}$ \\
\hline Total & 5730667 & 2989606 & 52,2 & 2741061 & 47,8 \\
\hline EU & 2392491 & 1256974 & 52,5 & 1135517 & 47,5 \\
\hline Resto de Europa & 234760 & 103306 & 44,0 & 131454 & 56,0 \\
\hline África & 1078899 & 678957 & 62,93 & 99942 & 37,1 \\
\hline América del Norte & 54547 & 24736 & 45,3 & 29811 & 54,7 \\
\hline América Central y Caribe & 206329 & 82328 & 39,9 & 124001 & 60,1 \\
\hline América del Sur & 1418751 & 636672 & 44,9 & 782079 & 55,1 \\
\hline Asia & 341886 & 204950 & 59,9 & 136.936 & 40,1 \\
\hline Resto & 3004 & 1683 & 56,0 & 1321 & 44,0 \\
\hline
\end{tabular}

Fuente: INE a 4 de abril de 2011. 